\title{
Comprehensive analysis of an IncRNA-miRNA-mRNA competing endogenous RNA network in pulpitis
}

\author{
Fangcao Lei ${ }^{1}$, Han Zhang ${ }^{2}$, Xiaoli Xie ${ }^{\text {Corresp. } 1}$ \\ ${ }^{1}$ Department of Operative Dentistry and Endodontics, School of Stomatology, Xiangya Stomatological Hospital, Central South University, Changsha, \\ Hunan, People's Republic of China \\ 2 Institute of Reproductive \& Stem Cell Engineering, School of Basic Medical Science, Central South University, Changsha, Hunan, People's Republic of \\ China \\ Corresponding Author: Xiaoli Xie \\ Email address: xiexiaoli@csu.edu.cn
}

Background. Pulpitis is a common inflammatory disease that affects dental pulp. It is important to understand the molecular signals of inflammation and repair associated with this process. Increasing evidence has revealed that long noncoding RNAs (IncRNAs), via competitively sponging microRNAs (miRNAs), can act as competing endogenous RNAs (ceRNAs) to regulate inflammation and reparative responses. The aim of this study was to elucidate the potential roles of IncRNA, miRNA and messenger RNA (mRNA) ceRNA networks in pulpitis tissues compared to normal control tissues.

Methods. The oligo and limma packages were used to identify differentially expressed IncRNAs and mRNAs (DElncRNAs and DEmRNAs, respectively) based on expression profiles in two datasets, GSE92681 and GSE77459, from the Gene Expression Omnibus (GEO) database. Differentially expressed genes (DEGs) were further analyzed by Gene Ontology (GO) and Kyoto Encyclopedia of Genes and Genomes (KEGG) pathway enrichment analyses. Protein-protein interaction (PPI) networks and modules were established to screen hub genes using the Search Tool for the Retrieval of Interacting Genes/Proteins (STRING) and the Molecular Complex Detection (MCODE) plugin for Cytoscape, respectively. Furthermore, an IncRNA-miRNA-mRNA-hub genes regulatory network was constructed to investigate mechanisms related to the progression and prognosis of pulpitis. Then, quantitative real-time polymerase chain reaction (qRT-PCR) was applied to verify critical IncRNAs that may significantly affect the pathogenesis in inflamed and normal human dental pulp.

Results. A total of 644 upregulated and 264 downregulated differentially expressed genes (DEGs) in pulpitis samples were identified from the GSE77459 dataset, while 8 up- and 19 downregulated probes associated with IncRNA were identified from the GSE92681 dataset. Protein-protein interaction (PPI) based on STRING analysis revealed a network of DEGs containing 4,929 edges and 623 nodes. Upon combined analysis of the constructed PPI network and the MCODE results, 10 hub genes, including IL6, IL8, PTPRC, IL1B, TLR2, ITGAM, CCL2, PIK3CG, ICAM1, and PIK3CD, were detected in the network. Next, a ceRNA regulatory relationship consisting of one IncRNA (PVT1), one miRNA ( hsa-miR-455-5p ) and two mRNAs (SOCS3 and PLXNC1) was established. Then, we constructed the network in which the regulatory relationship between ceRNA and hub genes was summarized. Finally, our qRT-PCR results confirmed significantly higher levels of PVT1 transcript in inflamed pulp than in normal pulp tissues $(p=0.03)$.

Conclusion. Our study identified a novel IncRNA-mediated ceRNA regulatory mechanisms in the pathogenesis of pulpitis. 
1 Comprehensive analysis of an IncRNA-miRNA-mRNA

2 competing endogenous RNA network in pulpitis

3

4

5

6

7

8

\section{Fangcao Lei ${ }^{1}$, Han Zhang ${ }^{2}$, Xiaoli Xie ${ }^{1}$}

${ }^{1}$ Department of Operative Dentistry and Endodontics, School of Stomatology, Xiangya

Stomatological Hospital, Central South University, Changsha, Hunan, People's Republic of China

${ }^{2}$ Institute of Reproductive \& Stem Cell Engineering, School of Basic Medical Science, Central South University, Changsha, Hunan, People's Republic of China

Corresponding Author:

Dr. Xiaoli Xie

No. 72 Xiangya Road, Changsha, Hunan, 410083, People's Republic of China Email address: xiexiaoli@csu.edu.cn

\section{Abstract}

Background. Pulpitis is a common inflammatory disease that affects dental pulp. It is important to understand the molecular signals of inflammation and repair associated with this process. Increasing evidence has revealed that long noncoding RNAs (lncRNAs), via competitively sponging microRNAs (miRNAs), can act as competing endogenous RNAs (ceRNAs) to regulate inflammation and reparative responses. The aim of this study was to elucidate the potential roles of lncRNA, miRNA and messenger RNA (mRNA) ceRNA networks in pulpitis tissues compared to normal control tissues.

Methods. The oligo and limma packages were used to identify differentially expressed lncRNAs and mRNAs (DElncRNAs and DEmRNAs, respectively) based on expression profiles in two datasets, GSE92681 and GSE77459, from the Gene Expression Omnibus (GEO) database. Differentially expressed genes (DEGs) were further analyzed by Gene Ontology (GO) and Kyoto Encyclopedia of Genes and Genomes (KEGG) pathway enrichment analyses. Protein-protein interaction (PPI) networks and modules were established to screen hub genes using the Search Tool for the Retrieval of Interacting Genes/Proteins (STRING) and the Molecular Complex Detection (MCODE) plugin for Cytoscape, respectively. Furthermore, an IncRNA-miRNAmRNA-hub genes regulatory network was constructed to investigate mechanisms related to the progression and prognosis of pulpitis. Then, quantitative real-time polymerase chain reaction (qRT-PCR) was applied to verify critical lncRNAs that may significantly affect the pathogenesis in inflamed and normal human dental pulp.

Results. A total of 644 upregulated and 264 downregulated differentially expressed genes (DEGs) in pulpitis samples were identified from the GSE77459 dataset, while 8 up- and 19 downregulated probes associated with lncRNA were identified from the GSE92681 dataset. 
40 Protein-protein interaction (PPI) based on STRING analysis revealed a network of DEGs

41 containing 4,929 edges and 623 nodes. Upon combined analysis of the constructed PPI network

42 and the MCODE results, 10 hub genes, including IL6, IL8, PTPRC, IL1B, TLR2, ITGAM,

43 CCL2, PIK3CG, ICAM1, and PIK3CD, were detected in the network. Next, a ceRNA regulatory

44 relationship consisting of one lncRNA (PVT1), one miRNA (hsa-miR-455-5p) and two mRNAs

45 (SOCS3 and PLXNC1) was established. Then, we constructed the network in which the

46 regulatory relationship between ceRNA and hub genes was summarized. Finally, our qRT-PCR

47 results confirmed significantly higher levels of PVT1 transcript in inflamed pulp than in normal

48 pulp tissues $(\mathrm{p}=0.03)$.

49 Conclusion. Our study identified a novel lncRNA-mediated ceRNA regulatory mechanisms in

50 the pathogenesis of pulpitis.

51

\section{Introduction}

53 Inflammation of the dental pulp (pulpitis) is associated with microbial infection of the root canal system and the host response, which is characterized by spontaneous or provoked pain (Bender, 2000; Rocas et al., 2015). Acute pulpitis can be an extremely painful condition and is believed to be one of the main reasons patients seek emergency dental treatment (Currie et al., 2017). Depending on the state of inflammation, various treatment regimens are currently applied clinically, including nonsurgical root canal treatment (such as pulpotomy and pulpectomy) and surgical endodontic treatment (European Society of Endodontology, 2006). If the issue cannot be solved in time or appropriately, pulpitis may progress to pulp necrosis, periapical periodontitis, or even severe oral and maxillofacial space infections (Bertossi et al., 2017), ultimately causing a significant medical and economic burden in terms of treatment costs.

Pulp inflammation represents a complex physiological response to harmful stimuli, such as bacterial infections and physical and chemical injuries (Yu \& Abbott, 2016; Larsen \& Fiehn, 2017; Pedano et al., 2018). The balance between inflammation and reparative processes in host defense reactions can determine the extent of pulp inflammation, in which multiple signaling pathways are involved (Cooper, Holder \& Smith, 2014; Farges et al., 2015). Studies over the past decade have provided much information on the role of epigenetic modifications in inflammatory diseases such as pulpitis and periodontitis. These studies have investigated modifications of histones, methylation of DNA, and regulation of noncoding RNAs (ncRNAs), among other epigenetic mechanisms (Kearney et al., 2018).

Due to the application of sequencing and bioinformatics approaches, recent evidence has identified previously unannotated transcripts and ncRNAs involved in pathologic mechanisms, such as microRNAs (miRNAs), long noncoding RNAs (lncRNAs), pseudogenes and circular RNAs, which were previously believed to have no biological functions (Kaikkonen \& Adelman, 2018). MiRNAs are small ( 21-24 nucleotides in length), single-stranded ncRNA molecules that can bind to complementary sequences within the 3' untranslated regions (UTRs) of target messenger RNAs (mRNAs), resulting in mRNA degradation or repression (Afonso-Grunz \& Muller, 2015). LncRNAs, which are ncRNAs of $>200$ nucleotides, function as competing 
80

81

82

83

84

85

86

87

88

89

90

91

92

93

94

95

96

97

98

99

100

101

102

103

104

105

106

107

108

109

110

111

112

113

114

115

116

117

118

119

endogenous RNAs (ceRNAs) that interact with mRNAs, serving as miRNA sponges to restrain miRNA function by competing for miRNA response elements (MREs) (Tay, Rinn \& Pandolfi, 2014). Increasing evidence has illustrated the essential roles of differentially expressed miRNAs and IncRNAs (DEmiRNAs and DElncRNAs, respectively) in a variety of cellular and pathologic processes associated with pulpitis, in which abnormal expression occurs (Zhong et al., 2012; Huang \& Chen, 2018). However, there have been few comprehensive analyses of pulpitisassociated lncRNAs and miRNAs in the context of a ceRNA network. The discovery of ceRNA network interactions could provide important insights to advance our understanding of the pathogenesis of pulpitis.

In this work, we first performed an integrated analysis and identified differentially expressed genes (DEGs) between pulpitis and matched normal pulp tissues from two gene expression profiles in the Gene Expression Omnibus (GEO) database. Then, Gene Ontology (GO) enrichment and Kyoto Encyclopedia of Genes and Genomes (KEGG) pathway analysis were further conducted to analyze the major biological functions of the DEGs. Next, we examined potential crosstalk in a constructed IncRNA-miRNA-mRNA ceRNA network that involved one core lncRNA, one miRNA, and two mRNAs. Moreover, ten hub genes related to pulpitis were identified as being aberrantly expressed in the protein-protein interaction (PPI) network. Ultimately, the DElncRNA plasmacytoma variant translocation gene 1(PVT1), which was significantly upregulated in inflamed pulp tissue, was validated by quantitative real-time polymerase chain reaction (qRT-PCR).To the best of our knowledge, this study is the first attempt to apply bioinformatics approaches to investigate the differential expression profiles of a specific lncRNA-mediated ceRNA network in inflamed pulp.

\section{Materials \& Methods}

\section{Microarray Data}

Microarray data from the GSE92681 and GSE77459 datasets were downloaded from the GEO (https://www.ncbi.nlm.nih.gov/geo) of NCBI. GSE92681 including 7 pulpitis and 5 matched normal pulp tissues was associated with the GPL16956 platform (Agilent-045997 Arraystar Human LncRNA Microarray V3) (Huang \& Chen, 2018). GSE77459 contained 6 pulpitis tissue specimens and 6 matched normal samples, and was associated the GPL17692 platform (Affymetrix Human Gene 2.1 ST Array) (Galicia et al., 2016). The DEmiRNAs were retrieved as reported by Zhong et al. (2012); until now, that data was the only known DEmiRNAs between normal and inflamed human pulp tissue. All data were freely accessible online.

\section{Data Processing}

The oligo package was used to read the microarray and normalize the expression data (Carvalho \& Irizarry, 2010). After the raw data were transformed to the expression files, the expression files were further processed with the Linear Models for Microarray data (limma) R/Bioconductor package (http://www.bioconductor.org) for analysis of the DEGs between pulpitis samples and 
120

121

122

123

124

125

126

127

128

129

130

131

132

133

134

135

136

137

138

139

140

141

142

143

144

145

146

147

148

149

150

151

152

153

154

155

156

157

158

159

control samples with biological replication; the Benjamin and Hochberg method was applied for multiple testing corrections (Ritchie et al., 2015). Genes that met the cutoff criteria, a corrected $p$-value $<0.05$ and a $\mid \log _{2}$ fold change $(\mathrm{FC}) \mid>1$, were considered DEGs.

\section{Function and Pathway Enrichment Analysis of the DEmRNAs}

To investigate pulpitis progression at the functional level, GO and pathway enrichment analyses of the identified DEmRNAs were performed in this study. The identified DEGs were enriched for terms in the GO biological process (BP), molecular function (MF), and cellular component (CC) categories (Ashburner et al., 2000). KEGG analysis was utilized to interpret the potential functions and pathways of the aberrantly expressed genes (Kanehisa et al., 2010). In addition, the above data were analyzed using the clusterProfiler package in R (Yu et al., 2012).

\section{Protein-Protein Interaction (PPI) Network Analysis}

The Search Tool for the Retrieval of Interacting Genes/Proteins (STRING, https://string-db.org/) was used to identify PPIs among the selected DEGs. A combined score $\geq 0.4$ was chosen for PPI network construction (Szklarczyk et al., 2015). Cytoscape software was used to visualize the established DEGs network, while the MCODE plugin and CentiScaPe in Cytoscape were used to select significant modules and calculate the connective of genes from the PPI network (Shannon et al., 2003; Scardoni et al., 2014).

\section{Construction of the ceRNA Network}

The lncRNA-associated ceRNA network in pulpitis was next constructed. First, miRNA-lncRNA interactions were evaluated using the starBase (version 2.0) database with the default parameters (clade: mammal, genome: human, assembly: hg19, number of supporting experiments: $\geq 1$ ) (Li et al., 2014). Next, starBase (version 2.0), TargetScan (release 7.1), and miRDB (last modified: May 03, 2016) were used to retrieve miRNA-targeted mRNAs (Li et al., 2014; Agarwal et al., 2015; Wong \& Wang, 2015). The main steps of the method are shown in Fig. 1.

\section{Validation Based on Clinical Samples of Human Dental Pulp}

To further verify the expression of the DElncRNAs, qRT-PCR was performed. Briefly, 4 human inflamed pulp tissue samples and 4 normal pulp tissue samples were obtained from Xiangya Stomatological Hospital. The normal pulp tissues were collected from healthy third molars extracted for orthodontic purposes. The inflamed pulp tissues were extracted from teeth diagnosed with irreversible pulpitis in accordance with the endodontic diagnosis system from the American Association of Endodontists. All patients gave written informed consent. The Ethics Committee of Xiangya Stomatological Hospital of Central South University granted Ethical approval to carry out the study within its facilities (Ethical Approval number: 20180026). Next, RNA from all tissue samples, which were previously preserved in liquid nitrogen, was extracted with TRIzol reagent (Thermo Fisher, USA). Reverse transcription was performed with a Transcriptor First Strand cDNA Synthesis Kit (Roche, USA). qRT-PCR was used to monitor the 
160

161

162

163

164

165

166

167

168

169

170

171

172

173

174

175

176

177

178

179

180

181

182

183

184

185

186

187

188

189

190

191

192

193

194

195

196

197

198

199

expression of PVT1 (Fw, 5' -TGAGAACTGTCCTTACGTGACC-3' ; Rev, 5' AGAGCACCAAGACTGGCTCT-3' ) and GAPDH (Fw, 5' -

GACAGTCAGCCGCATCTTCTT-3' ; Rev, 5' -AATCCGTTGACTCCGACCTTC-3' ); GAPDH was used as the housekeeping gene for normalization. Amplification was performed with LightCycler 480 SYBR Green I Master (Roche, USA) on a real-time PCR system (LightCycler 480, Roche, USA). Threshold cycle values (CT) were determined, and the data were analyzed with Roche software with the $2^{-\Delta \Delta C T}$ method. An unpaired Wilcoxon test was conducted to compare lncRNA expression at the transcriptional level between normal and inflamed pulp tissues.

\section{Results}

\section{Identification of DEGs in Pulpitis}

Based on the criteria of $p<0.05$ and $\left|\log _{2} \mathrm{FC}\right|>1$, a total of 908 DEGs were screened from GSE77459, including 264 downregulated genes and 644 upregulated genes in pulpitis. In the GSE92681 dataset, 27 probes associated with DElncRNA, including 19 downregulated and 8 upregulated probes, were identified. A volcano plot for GSE77459 and a heatmap for GSE92681 are shown in Fig. 2. The detailed differential expression profiles are summarized in Supplementary file 1 .

\section{GO and KEGG Analysis of the DEGs}

GO function and KEGG pathway enrichment analyses of the DEGs were performed using the clusterProfiler package in $\mathrm{R}$. The results of GO analysis illustrated that the DEGs were enriched for BP terms including leukocyte migration, the adaptive immune response, the immune response-regulating cell surface receptor signaling pathway, regulation of leukocyte activation, and the immune response-activating cell surface receptor signaling pathway. CC analysis showed that the DEGs were significantly enriched for the side of membrane, secretory granule membrane, and external side of plasma membrane, tertiary granule, and specific granule terms. For the MF category, the DEGs were enriched in antigen binding, serine-type peptidase activity, serine hydrolase activity, serine-type endopeptidase activity, and cytokine activity (Fig. 3).

Based on KEGG pathway analysis, the DEGs were significantly enriched in pathways associated with cytokine-cytokine receptor interaction, the chemokine signaling pathway, cell adhesion molecules, Staphylococcus aureus infection, and the hematopoietic cell lineage (Fig. 4). The detailed results of the GO enrichment and KEGG pathway analyses are provided in Supplementary file 2 .

\section{PPI Network Construction and Hub Genes Identification}

Protein interactions among the DEGs were detected with the online STRING program with a cutoff score of $\geq 0.4$. In total, 4,929 edges and 623 nodes were involved in the PPI network. One significant module containing 528 edges and 33 nodes was selected from the PPI network by the 
200 MCODE plugin in Cytoscape (Fig. 5). In this module, we found that most of the genes were

201

202

203

204

205

206

207

208

209

210

211

212

213

214

215

216

217

218

219

220

221

222

223

224

225

226

227

228

229

230

231

232

233

234

235

236

237

238

239

mainly enriched for and associated with chemokines (Tables 1 and 2).

In addition, we screened the top 10 hub mRNAs from the PPI network. These main hub genes were IL6, IL8, PTPRC, IL1B, TLR2, ITGAM, CCL2, PIK3CG, ICAM1, and PIK3CD. Hence, the key genes associated with pulpitis could be predicted by our network.

\section{Construction and Analysis of the ceRNA Network in Pulpitis}

To identify correlations among the DElncRNAs in the ceRNA network, we used starBase to search for interactions between miRNAs and lncRNAs. The target mRNAs of the miRNAs in the network were predicted with starBase, TargetScan, and miRDB. The ceRNA network was constructed based on coexpressed lncRNAs/miRNAs, miRNAs/mRNAs, and lncRNAs/mRNAs. Overlapping datasets were visualized using Venn diagrams (Fig. 6A). As shown in Fig. 6B, the lncRNA-miRNA-mRNA network was composed of one lncRNA (PVT1), one miRNA (hsamiR-455-5p), and two mRNAs (suppressor of cytokine signaling 3, SOCS3 and Plexin C1, PLXNC1). In addition, a comprehensive analysis of the relationships among PVT1, hsa-miR455-5p, SOCS3 and PLXNC1 and the top 10 downstream connected genes is depicted in Fig. $6 \mathrm{~B}$.

\section{Validation of PVT1 expression in Clinical Samples of Human Dental Pulp}

We evaluated the expression of PVT1 by qRT-PCR in pulpitis tissues compared to normal pulp tissues. As depicted in Fig. 7, PVT1 transcript levels were significantly higher in inflamed pulp than in normal pulp $(\mathrm{p}<0.05)$.

\section{Discussion}

Pulpitis is considered a tightly regulated process involving microorganisms and host immune events mediated by molecular factors (Renard et al., 2016). Inflammation of the dental pulp is characterized by opportunistic infection of the pulp space by commensal oral microorganisms, such as Porphyromonas and Streptococcus species (Rocas et al., 2016). The most common route for microorganism invasion is through dental caries. Other possible portals include tooth damage from trauma, exposed dentinal tubules or the apical foramen (Raslan \& Wetzel, 2006). Upon irritation, cells in human dental pulp, for example, endothelial cells, odontoblasts and macrophages, immediately trigger immune responses to pathogens and their virulent factors, potentially stalling the spread of infection, preventing injury-related signaling, and launching reparative processes (Rechenberg, Galicia \& Peters, 2016). However, if the delicate balance between the immune-inflammatory response and dental tissue healing is disrupted, irreversible pulpitis could result from uncontrollable inflammation caused by the invading bacteria.

Over the past decades, ncRNAs, including lncRNAs and short ncRNAs (such as miRNAs), have gained increasing attention for their roles in physiological and pathologic responses. In diverse biological processes, gene expression is regulated by posttranscriptional mechanisms involving ncRNAs through their binding to the 3'-UTRs of target mRNAs, leading 
240 to translational repression or target degradation (Vasudevan, 2012). Accumulating studies have 241 provided evidence supporting the ceRNA hypothesis, which holds that lncRNAs harboring 242 MREs can competitively bind to certain miRNAs, thus regulating miRNA-mediated downstream 243 target gene silencing at the posttranscriptional level (Khorkova, Hsiao \& Wahlestedt, 2015).

244 Although the functional relevance of several lncRNAs and miRNAs in pulpitis has been proven 245 in the scientific literature, an lncRNA-based ceRNA network involved in pulpitis has yet to be 246 defined (Zhong et al., 2012; Huang \& Chen, 2018). In the current study, bioinformatics analyses 247 were applied to explore the expression profiles of lncRNAs, miRNAs, and mRNAs in patients 248 with pulpitis. Furthermore, an lncRNA-related ceRNA network was constructed by integrating 249 data from GEO pulpitis expression profiles. These data might shed light on how lncRNA250 miRNA-mRNA interactions are involved in pulpitis. Immune response can be triggered by 251 inflammatory mediators or cytokines produced by infectious cells through the transcriptional or 252 post-transcriptional regulations. The expression of ncRNAs may be closely related to the development of immune cells (particularly monocytes, macrophages, NK cells, T helper cells, $\mathrm{CD}^{+} \mathrm{T}$ cells, and Treg cells) and the maintenance of immune system homeostasis (Cortez et al., 2019; Imamura \& Akimitsu, 2014). For instance, lincRNA-Cox2 regulates the release of

257

258

259

260

261

262

263

264

265

266

267

268

269

270

271

272

273

274

275

276

277

278

279 inflammatory mediators and cytokines by stimulation of TLR2 in bacterial infections (Carpenter et al. 2013), and miR-155 exerts an antiproliferative effect on $\mathrm{CD}^{+} \mathrm{T}$ cells in response to the type I interferon signaling (Gracias et al., 2013).

LncRNAs and miRNAs, which are closely correlated with inflammatory mediator production and pulp repair, could be affected by the inflammatory status in dental pulp (Zhong et al., 2012; Galicia et al., 2016; Huang \& Chen, 2018). In this study, our findings indicated that lncRNA PVT1might regulate the progression of pulpitis by acting as a sponge for miR-455-5p. Emerging studies have proven that PVT1 plays an important role in inflammation and tumorigenesis. For example, down-regulation of PVT1 correlates with the differentiation of Th17 cells and the duration of multiple sclerosis, a chronic immune-mediated disease (Eftekharian et al., 2017). Additionally, PVT1 promotes the production of inflammatory cytokines to aggravate the progression of IL-1 $\beta$-stimulated osteoarthritis (Zhao et al., 2018). Overexpressed PVT1 can enhance the expression of IL-6 and IL-1 $\beta$ in a nonbinding manner by regulating the nuclear factor $-\kappa \mathrm{B}(\mathrm{NF}-\kappa \mathrm{B})$ pathway, which is considered a critical mechanism in the regulation of immune and inflammatory processes, and ultimately aggravating septic acute kidney injury (Huang et al., 2017). Moreover, some studies have revealed that PVT1, which is located on 8q24.21 and contains the myc proto-oncogene, is upregulated in certain human tumors (Shtivelman \& Bishop, 1989) and is correlated with pathologic stage in multiple cancers (Chai et al., 2018). In this study, our qRT-PCR results indicated that PVT1 was upregulated in pulpitis tissue compared with matched normal pulp tissue, suggesting that PVT1 is an important factor contributing to pulpitis. However, the exact role of PVT1 in the occurrence and development of pulp inflammation and the underlying mechanism require further investigation.

MiR-455-5p is one subtype of the mature miR-455, which is located in the sense strand of chromosome 9q32, and is a tumor-associated miRNA molecule. The other subtype is miR- 
280

281

282

283

284

285

286

287

288

289

290

291

292

293

294

295

296

297

298

299

300

301

302

303

304

305

306

307

308

309

310

311

312

313

314

315

316

317

318

319

455-3p (Arai et al., 2019). MiR-455 is involved in a variety of biological processes through the repression of specific mRNA at the posttranscriptional level. For example, miR-455-5p regulates the posttranscriptional repression of UDP-glucuronosyltransferase (UGT) 2B expression by binding to MREs in 3'-UTR of UGT2B, which plays a role in drug glucuronidation (Papageorgiou \& Court, 2017). Moreover, in colorectal cancer, miR-455 is up-regulated to inhibit the protein expression of RAF proto-oncogene serine/threonine protein kinase (RAF1) other than affects mRNA level, which regulates the cellular proliferation and invasion (Chai et al., 2015). In pulpitis, the precise posttranscriptional mechanisms of miR-455-5p remain to be explored. Previous studies have revealed that miR-455-5p can serve as a prognostic biomarker and therapeutic target for patients with certain cancers, such as non-small cell lung cancer and basal cell carcinoma (Sand et al., 2012; Wang et al., 2017). Moreover, miR-455 represents novel regulator of the immune and inflammatory response.MiR-455-5p plays an anti-inflammatory role in multiple sclerosis. MiR-455-5p is negatively correlated with the activation of the NF- $\mathrm{kB}$ pathway, thus inhibiting the expression of IL-1 $\beta$, IL- 6 and IL- 8 and in turn ameliorating the severity of multiple sclerosis (Torabi et al., 2019). Macrophage polarization is an essential component of immunity and homeostasis, and miR-455-5p overexpression reverses the polarization of macrophages to the M1 phenotype to reduce the secretion of proinflammatory cytokines, such as IL-1 $\beta$ and TNF- $\alpha$ (Chi et al., 2018). The accumulation of miR-455 regulates the innate immune response in E2F1-deficient mice, leading to reduced inflammation induced by LPS (Warg et al., 2012). In patients infected with the hepatitis B virus, serum miR-455-3p levels are decreased during chronic disease progression (Singh et al., 2018). The algorithms in our study predicted that miR-455-5p was downregulated (Fig. 6B) in pulpitis samples compared to control samples. Further work will be needed with multiple clinical samples to clarify the action of miR-455-5p in pulpitis and thus to uncover the mechanisms underlying the effects of miR$455-5 \mathrm{p}$ on proinflammatory processes.

Based on the module produced by the STRING analysis, two core mRNAs, identified as SOCS3 and PLXNC1, can be considered important target genes of miR-455-5p. SOCS3 is a major suppressor of inflammation and is known as a feedback inhibitor of the JAK/STAT3 signaling pathway (Carow \& Rottenberg, 2014). SOCS3 can inhibit JAK2 activity to attenuate STAT3 phosphorylation, inhibit the NF- $\mathrm{KB}$ pathway and trigger the expression of various genes in response to cytokines (the IL-6 family and IL-10), consequently affecting cell proliferation, differentiation, and apoptosis (Gao et al., 2018). SOCS3 deficiency results in increased alveolar loss with high levels of IL-1 $\beta$, IL-6, and IL-8 in periodontitis (Papathanasiou et al., 2016). Furthermore, induction of SOCS3 expression may markedly reduce cell adherence by inhibiting TNF- $\alpha$-stimulated ICAM1 expression in lung inflammation (Lee et al., 2013). PLXNC1 is an endogenous receptor of the neuronal guidance protein semaphorin 7a (Sema 7a). Previous studies have suggested that PLXNC1 might play an important role in immunological and proinflammatory responses. A study by Konig et al. (2014) on PLXNC1 ${ }^{-/}$mice provided evidence that PLXNC1 depletion results in a reduced inflammatory response and decreased cytokine IL-1 $\beta$ production in vivo. We hypothesize that PLXNC1 and SOCS3 play important 
320

321

322

323

324

325

326

327

328

329

330

331

332

333

334

335

336

337

338

339

340

341

342

343

344

345

346

347

348

349

350

351

352

353

354

355

356

357

358

359

roles in conditions associated with inflammation. Both might therefore be potential targets to prevent damage due to excessive tissue inflammation.

GO and pathway analyses were used to infer the potential functions of the DEGs in pulpitis. GO annotation revealed that the top GO terms in the BP and MF categories for the DEGs between the inflamed and healthy pulp tissue were mainly associated with the immune/inflammation system (Fig. 3), suggesting that the cascade of cellular events in pulpitis is precisely regulated by migrating leukocytes and their surrounding microenvironments and also supporting other previous theories explaining the occurrence of pulpitis (Cooper et al., 2017). Furthermore, KEGG pathway analysis indicated that the cytokine-cytokine receptor interaction pathway was the most highly enriched pathway for the upregulated lncRNAs between pulpitis and normal dental tissues, while other classic inflammatory signaling pathways, including the chemokine signaling pathway, cell adhesion molecules (CAMs), Staphylococcus aureus infection, the hematopoietic cell lineage, the TNF signaling pathway, and osteoclast differentiation were also enriched. These pathways were mainly involved in immune regulation, intercellular signaling, defense mechanisms, osteoclast differentiation, and hematopoiesis.

\section{Conclusions}

To the best of our knowledge, there have been no studies on lncRNA-associated ceRNA networks in pulpitis. Here, we used bioinformatics methods to elucidate the IncRNA-miRNAmRNA ceRNA network associated with dental pulp inflammation. Our analysis suggests a potential mechanism by which PVT1 competes with miR-455-5p to regulate the expression of SOCS3 and PLXNC1. Upregulation of PVT1, which has been validated in inflamed pulp by qRT-PCR, could increase inflammation and cytokine and chemokine production in inflamed pulp tissue compared with healthy pulp tissue. In addition, overexpression of PVT1 reduced miR-455-5p expression and indirectly promoted SOCS3 and PLXNC1 expression and the cytokine cascade. In addition, SOCS3 and PLXNC1 could regulate the hub genes (including IL$1 \beta$, IL-6, IL-8 and ICAM1), which play a critical role in pulp inflammation. Upon comprehensive analysis of the lncRNA-related ceRNA network, some novel and crucial characteristics of pulpitis were revealed. These findings provide new insights into the pathogenesis of endodontic lesions and might identify potential diagnostic and therapeutic strategies for future studies.

\section{References}

Afonso-Grunz F, Muller S. 2015. Principles of miRNA-mRNA interactions: beyond sequence complementarity. Cellular and Molecular Life Sciences 72:3127-3141 DOI: 10.1007/s00018-015-1922-2.

Agarwal V, Bell GW, Nam JW, Bartel DP. 2015. Predicting effective microRNA target sites in mammalian mRNAs. Elife 4: e05005 DOI: 10.7554/eLife.05005.

Arai T, Kojima S, Yamada Y, Sugawara S, Kato M, Yamazaki K, Naya Y, Ichikawa T, Seki N. 2019. Pirin: a potential novel therapeutic target for castration-resistant prostate cancer 
360

361

362

363

364

365

366

367

368

369

370

371

372

373

374

375

376

377

378

379

380

381

382

383

384

385

386

387

388

389

390

391

392

393

394

395

396

397

398

regulated by miR-455-5p. Molecular Oncology 13:322-337 DOI: 10.1002/18780261.12405 .

Ashburner M, Ball CA, Blake JA, Botstein D, Butler H, Cherry JM, Davis AP, Dolinski K, Dwight SS, Eppig JT, Harris MA, Hill DP, Issel-Tarver L, Kasarskis A, Lewis S, Matese JC, Richardson JE, Ringwald M, Rubin GM, Sherlock G. 2000. Gene ontology: tool for the unification of biology. The gene ontology consortium. Nature Genetics 25:25-29 DOI: $10.1038 / 75556$.

Bender IB. 2000. Pulpal pain diagnosis--a review. Journal of Endodontics 26:175-179 DOI: 10.1097/00004770-200003000-00012.

Bertossi D, Barone A, Iurlaro A, Marconcini S, De Santis D, Finotti M, Procacci P. 2017. Odontogenic orofacial infections. The Journal of Craniofacial Surgery 28:197-202 DOI: $10.1097 /$ scs.0000000000003250.

Carow B, Rottenberg ME. 2014. SOCS3, a major regulator of infection and inflammation. Frontiers in Immunology 5:58 DOI: 10.3389/fimmu.2014.00058.

Carvalho BS, Irizarry RA. 2010. A framework for oligonucleotide microarray preprocessing. Bioinformatics (Oxford, England) 26:2363-2367 DOI: 10.1093/bioinformatics/btq431.

Chai J, Guo D, Ma W, Han D, Dong W, Guo H, Zhang Y. 2018. A feedback loop consisting of RUNX2/LncRNA-PVT1/miR-455 is involved in the progression of colorectal cancer. American Journal of Cancer Research 8:538-550.

Chai J, Wang S, Han D, Dong W, Xie C, Guo H. 2015. MicroRNA-455 inhibits proliferation and invasion of colorectal cancer by targeting RAF proto-oncogene serine/threonine-protein kinase. Tumour Biology 36:1313-1321 DOI: 10.1007/s13277-014-2766-3.

Chi X, Ding B, Zhang L, Zhang J, Wang J, Zhang W. 2018. IncRNA GAS5 promotes M1 macrophage polarization via miR-455-5p/SOCS3 pathway in childhood pneumonia. Journal of cellular physiology 234:13242-13251 DOI: 10.1002/jcp.27996.

Cooper PR, Chicca IJ, Holder MJ, Milward MR. 2017. Inflammation and regeneration in the dentin-pulp complex: net gain or net loss? Journal of Endodontics 43:S87-S94 DOI: 10.1016/j.joen.2017.06.011.

Cooper PR, Holder MJ, Smith AJ. 2014. Inflammation and regeneration in the dentin-pulp complex: a double-edged sword. Journal of Endodontics 40:S46-S51 DOI: 10.1016/j.joen.2014.01.021.

Cortez MA, Anfossi S, Ramapriyan R, Menon H, Atalar SC, Aliru M, Welsh J, Calin GA. 2019. Role of miRNAs in immune responses and immunotherapy in cancer. Genes Chromosomes Cancer 58:244-253 DOI: 10.1002/gcc.22725.

Currie CC, Stone SJ, Connolly J, Durham J. 2017. Dental pain in the medical emergency department: a cross-sectional study. Journal of Oral Rehabilitation 44:105-111 DOI: 10.1111/joor.12462.

Eftekharian MM, Ghafouri-Fard S, Soudyab M, Omrani MD, Rahimi M, Sayad A, Komaki A, Mazdeh M, Taheri M. 2017. Expression Analysis of Long Non-coding RNAs in the 
Blood of Multiple Sclerosis Patients. Journal of Molecular Neuroscience 63:333-341 DOI: $10.1007 / \mathrm{s} 12031-017-0982-1$.

Farges JC, Alliot-Licht B, Renard E, Ducret M, Gaudin A, Smith AJ, Cooper PR. 2015. Dental pulp defence and repair mechanisms in dental caries. Mediators of Inflammation 2015:230251 DOI: 10.1155/2015/230251.

Galicia JC, Henson BR, Parker JS, Khan AA. 2016. Gene expression profile of pulpitis. Genes and Immunity 17:239-243 DOI: 10.1038/gene.2016.14.

Gao Y, Zhao H, Wang P, Wang J, Zou L. 2018. The roles of SOCS3 and STAT3 in bacterial infection and inflammatory diseases. Scandinavian Journal of Immunology 88:e12727 DOI: $10.1111 /$ sji.12727.

Gracias DT, Stelekati E, Hope JL, Boesteanu AC, Doering TA, Norton J, Mueller YM, Fraietta JA, Wherry EJ, Turner M, Katsikis PD. 2013. The microRNA miR-155 controls CD8(+) $\mathrm{T}$ cell responses by regulating interferon signaling. Nature immunology 14:593-602 DOI: 10.1038/ni.2576.

Huang W, Lan X, Li X, Wang D, Sun Y, Wang Q, Gao H, Yu K. 2017. Long non-coding RNA PVT1 promote LPS-induced septic acute kidney injury by regulating TNFalpha and JNK/NF-kappaB pathways in HK-2 cells. International Immunopharmacology 47:134140 DOI: 10.1016/j.intimp.2017.03.030.

Huang X, Chen K. 2018. Differential expression of long noncoding RNAs in normal and inflamed human dental pulp. Journal of Endodontics 44:62-72 DOI: 10.1016/j.joen.2017.08.022.

Imamura K, and Akimitsu N. 2014. Long Non-Coding RNAs Involved in Immune Responses. Frontiers in immunology 5:573 DOI: 10.3389/fimmu.2014.00573.

Kaikkonen MU, Adelman K. 2018. Emerging roles of non-coding RNA transcription. Trends in Biochemical Sciences 43:654-667 DOI: https://doi.org/10.1016/j.tibs.2018.06.002.

Kanehisa M, Goto S, Furumichi M, Tanabe M, Hirakawa M. 2010. KEGG for representation and analysis of molecular networks involving diseases and drugs. Nucleic Acids Research 38:D355-D360 DOI: 10.1093/nar/gkp896.

Kearney M, Cooper PR, Smith AJ, Duncan HF. 2018. Epigenetic approaches to the treatment of dental pulp inflammation and repair: opportunities and obstacles. Frontiers in Genetics 9:311 DOI: 10.3389/fgene.2018.00311.

Khorkova O, Hsiao J, Wahlestedt C. 2015. Basic biology and therapeutic implications of IncRNA. Advanced Drug Delivery Reviews 87:15-24 DOI: 10.1016/j.addr.2015.05.012.

Konig K, Marth L, Roissant J, Granja T, Jennewein C, Devanathan V, Schneider M, Kohler D, Zarbock A, Rosenberger P. 2014. The plexin C1 receptor promotes acute inflammation. European Journal of Immunology 44:2648-2658 DOI: 10.1002/eji.201343968.

Larsen T, Fiehn NE. 2017. Dental biofilm infections - an update. Apmis 125:376-384 DOI: 10.1111/apm.12688.

Lee IT, Lin CC, Lee CY, Hsieh PW, Yang CM. 2013. Protective effects of (-)-epigallocatechin3-gallate against TNF-alpha-induced lung inflammation via ROS-dependent ICAM-1 
439

440

441

442

443

444

445

446

447

448

449

450

451

452

453

454

455

456

457

458

459

460

461

462

463

464

465

466

467

468

469

470

471

472

473

474

475

476

477

inhibition. The Journal of Nutritional Biochemistry 24:124-136 DOI:

10.1016/j.jnutbio.2012.03.009.

Li JH, Liu S, Zhou H, Qu LH, Yang JH. 2014. StarBase v2.0: decoding miRNA-ceRNA, miRNA-ncRNA and protein-RNA interaction networks from large-scale CLIP-Seq data. Nucleic Acids Research 42:D92-D97 DOI: 10.1093/nar/gkt1248.

European Society of Endodontology. 2006. Quality guidelines for endodontic treatment: consensus report of the European Society of Endodontology. International Endodontic Journal 39:921-930.

Papageorgiou I, and Court MH. 2017. Identification and validation of the microRNA response elements in the 3'-untranslated region of the UDP glucuronosyltransferase (UGT) 2B7 and 2B15 genes by a functional genomics approach. Biochemical Pharmacology 146:199-213 DOI: 10.1016/j.bcp.2017.09.013.

Papathanasiou E, Kantarci A, Konstantinidis A, Gao H, Van Dyke TE. 2016. SOCS-3 regulates alveolar bone loss in experimental periodontitis. Journal of Dental Research 95:10181025 DOI: 10.1177/0022034516645332.

Pedano MS, Li X, Li S, Sun Z, Cokic SM, Putzeys E, Yoshihara K, Yoshida Y, Chen Z, Van Landuyt K, Van Meerbeek B. 2018. Freshly-mixed and setting calcium-silicate cements stimulate human dental pulp cells. Dental Materials 34:797-808 DOI: 10.1016/j.dental.2018.02.005.

Raslan N, Wetzel WE. 2006. Exposed human pulp caused by trauma and/or caries in primary dentition: a histological evaluation. Dental Traumatology 22:145-153 DOI: 10.1111/j.1600-9657.2006.00410.x.

Rechenberg DK, Galicia JC, Peters OA. 2016. Biological markers for pulpal inflammation: a systematic review. PLoS One 11:e0167289 DOI: 10.1371/journal.pone.0167289.

Renard E, Gaudin A, Bienvenu G, Amiaud J, Farges JC, Cuturi MC, Moreau A, Alliot-Licht B. 2016. Immune cells and molecular networks in experimentally induced pulpitis. Journal of Dental Research 95:196-205 DOI: 10.1177/0022034515612086.

Ritchie ME, Phipson B, Wu D, Hu Y, Law CW, Shi W, Smyth GK. 2015. Limma powers differential expression analyses for RNA-sequencing and microarray studies. Nucleic Acids Research 43:e47 DOI: 10.1093/nar/gkv007.

Rocas IN, Alves FR, Rachid CT, Lima KC, Assuncao IV, Gomes PN, Siqueira JF, Jr. 2016. Microbiome of deep dentinal caries lesions in teeth with symptomatic irreversible pulpitis. PLoS One 11:e0154653 DOI: 10.1371/journal.pone.0154653.

Rocas IN, Lima KC, Assuncao IV, Gomes PN, Bracks IV, Siqueira JF, Jr. 2015. Advanced caries microbiota in teeth with irreversible pulpitis. Journal of Endodontics 41:1450-1455 DOI: 10.1016/j.joen.2015.05.013.

Sand M, Skrygan M, Sand D, Georgas D, Hahn SA, Gambichler T, Altmeyer P, Bechara FG. 2012. Expression of microRNAs in basal cell carcinoma. The British Journal of Dermatology 167:847-855 DOI: 10.1111/j.1365-2133.2012.11022.x.

Peer) reviewing PDF | (2019:02:35140:1:1:NEW 25 Apr 2019) 
478 Scardoni G, Tosadori G, Faizan M, Spoto F, Fabbri F, Laudanna C. 2014. Biological network

479

480

481

482

483

484

485

486

487

488

489

490

491

492

493

494

495

496

497

498

499

500

501

502

503

504

505

506

507

508

509

510

511

512

513

514

515

516

517 analysis with CentiScaPe: centralities and experimental dataset integration. F1000Research 3:139 DOI: 10.12688/f1000research.4477.2.

Shannon P, Markiel A, Ozier O, Baliga NS, Wang JT, Ramage D, Amin N, Schwikowski B, Ideker T. 2003. Cytoscape: a software environment for integrated models of biomolecular interaction networks. Genome Research 13:2498-2504 DOI: 10.1101/gr.1239303.

Shtivelman E, Bishop JM. 1989. The PVT gene frequently amplifies with MYC in tumor cells. Molecular and Cellular Biology 9:1148-1154.

Singh AK, Rooge SB, Varshney A, Vasudevan M, Bhardwaj A, Venugopal SK, Trehanpati N, Kumar M, Geffers R, Kumar V,Sarin SK. 2018. Global microRNA expression profiling in the liver biopsies of hepatitis B virus-infected patients suggests specific microRNA signatures for viral persistence and hepatocellular injury. Hepatology 67:1695-1709 DOI: 10.1002/hep.29690.

Szklarczyk D, Franceschini A, Wyder S, Forslund K, Heller D, Huerta-Cepas J, Simonovic M, Roth A, Santos A, Tsafou KP, Kuhn M, Bork P, Jensen LJ, von Mering C. 2015. STRING v10: protein-protein interaction networks, integrated over the tree of life. Nucleic Acids Research 43:D447-D452 DOI: 10.1093/nar/gku1003.

Tay Y, Rinn J, Pandolfi PP. 2014. The multilayered complexity of ceRNA crosstalk and competition. Nature 505:344-352 DOI: 10.1038/nature12986.

Torabi S, Tamaddon M, Asadolahi M, Shokri G, Tavakoli R, Tasharrofi N, Rezaei R, Tavakolpour V, Sazegar H, Kouhkan F. 2019. miR-455-5p downregulation promotes inflammation pathways in the relapse phase of relapsing-remitting multiple sclerosis disease. Immunogenetics 71:87-95 DOI: 10.1007/s00251-018-1087-x.

Vasudevan S. 2012. Posttranscriptional upregulation by microRNAs. Wiley Interdisciplinary Reviews. RNA 3:311-330 DOI: 10.1002/wrna.121.

Wang J, Wang Y, Sun D, Bu J, Ren F, Liu B, Zhang S, Xu Z, Pang S, Xu S. 2017. miR-455-5p promotes cell growth and invasion by targeting SOCO3 in non-small cell lung cancer. Oncotarget 8:114956-114965 DOI: 10.18632/oncotarget.22565.

Warg LA, Oakes JL, Burton R, Neidermyer AJ, Rutledge HR, Groshong S, Schwartz DA, Yang IV. 2012. The role of the E2F1 transcription factor in the innate immune response to systemic LPS. American journal of physiology. Lung cellular and molecular physiology 303:L391-400 DOI: 10.1152/ajplung.00369.2011.

Wong N, Wang X. 2015. miRDB: an online resource for microRNA target prediction and functional annotations. Nucleic Acids Research 43:D146-D152 DOI: 10.1093/nar/gku1104.

Yu CY, Abbott PV. 2016. Responses of the pulp, periradicular and soft tissues following trauma to the permanent teeth. Australian Dental Journal 61 Suppl 1:39-58 DOI: 10.1111/adj.12397.

Yu G, Wang LG, Han Y, He QY. 2012. ClusterProfiler: an R package for comparing biological themes among gene clusters. OMICS 16:284-287 DOI: 10.1089/omi.2011.0118. 
518 Zhao Y, Zhao J, Guo X, She J, Liu Y. 2018. Long non-coding RNA PVT1, a molecular sponge 519 for miR-149, contributes aberrant metabolic dysfunction and inflammation in IL-1betasimulated osteoarthritic chondrocytes. Bioscience Reports 38:BSR20180576 DOI:

$521 \quad 10.1042 / \mathrm{bsr} 20180576$.

522 Zhong S, Zhang S, Bair E, Nares S, Khan AA. 2012. Differential expression of microRNAs in 523 normal and inflamed human pulps. Journal of Endodontics 38:746-752 DOI:

$524 \quad$ 10.1016/j.joen.2012.02.020. 
Figure 1 (on next page)

Main steps of the construction of the regulatory network in pulpitis.

Step 1: We identified the differentially expressed mRNAs, IncRNAs and miRNAs. Step 2: The GO and KEGG enrichment analyses were conducted and the PPI network was constructed. Then, we identified the hub genes from the PPI network. Step 3: The ceRNA regulatory relationships were predicted using online tools. Step 4: The ceRNA network with hub genes was constructed. 


\section{Figure 2}

DEGs between pulpits samples and normal samples.

(A) Volcano plot for the DEGs in dataset GSE77459. The x-axis indicates the log FC, and the $y$-axis indicates the log10 (adjusted p-value). The red dots represent upregulated genes, and the blue dots represent downregulated genes. The DEGs were screened on the basis of a |fold change| $>1.0$ and an adjusted $p$-value of $<0.05$. The black dots represent genes with no significant difference. FC, fold change. (B) Heatmap of the DEGs in dataset GSE92681. The relative expression values were normalized to fall in a range from zero to one. Genes expressed at high levels are shown in blue, while those expressed at low levels are shown in white.

A

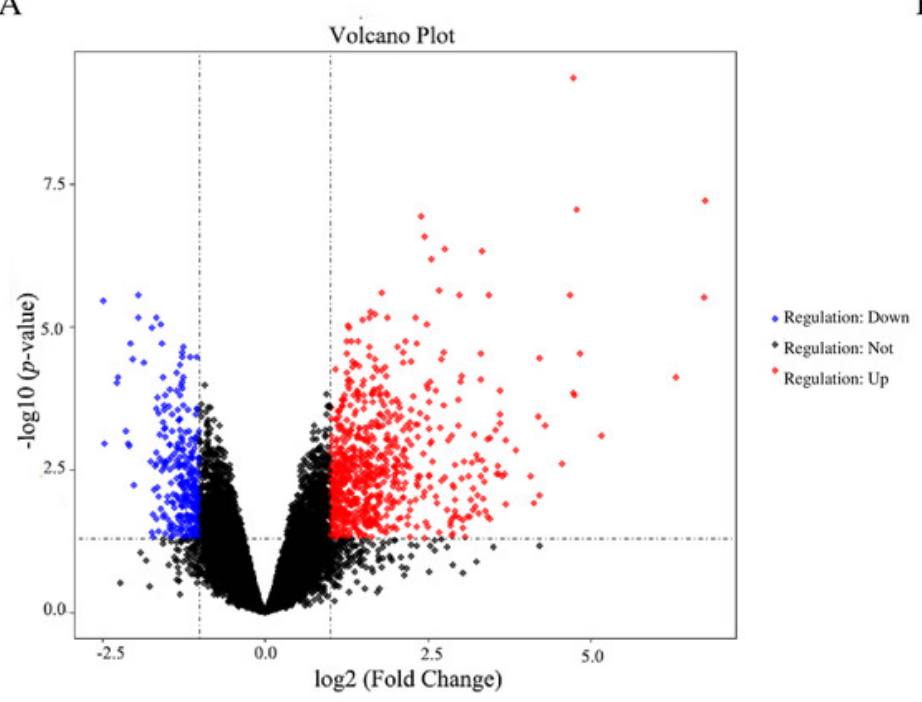

B

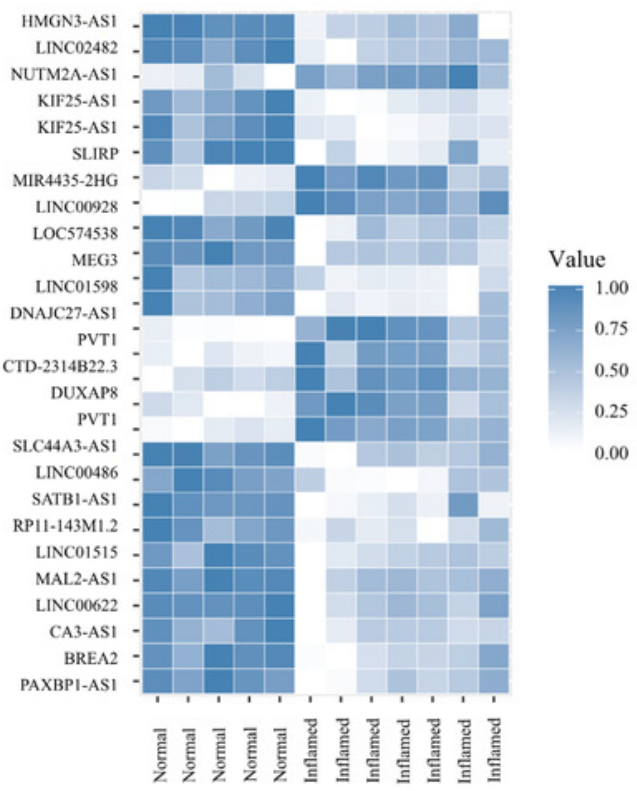


Figure 3 (on next page)

Top 10 processes revealed in GO enrichment to influence biological process (BP), molecular function (MF), and cellular component (CC).

The colored dots represent the term enrichment: blue indicates low enrichment, and red indicates high enrichment. The sizes of the dots represent the numbers of genes in each GO category. 
Figure 4 (on next page)

Top 10 enriched KEGG pathways for the upregulated DEGs.

The $y$-axis shows the KEGG pathway names. The colored dots represent the term

enrichment: blue indicates low enrichment, while red indicates high enrichment. The sizes of the dots represent the numbers of genes. 
Cytokine-cytokine receptor interaction

Chemokine signaling pathway Cell adhesion molecules (CAMs)

Staphylococcus aureus infection

Hematopoietic cell lineage

TNF signaling pathway

Osteoclast differentiation Rheumatoid

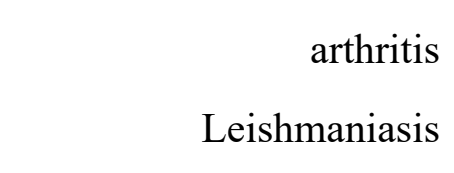

Graft-versus-host disease

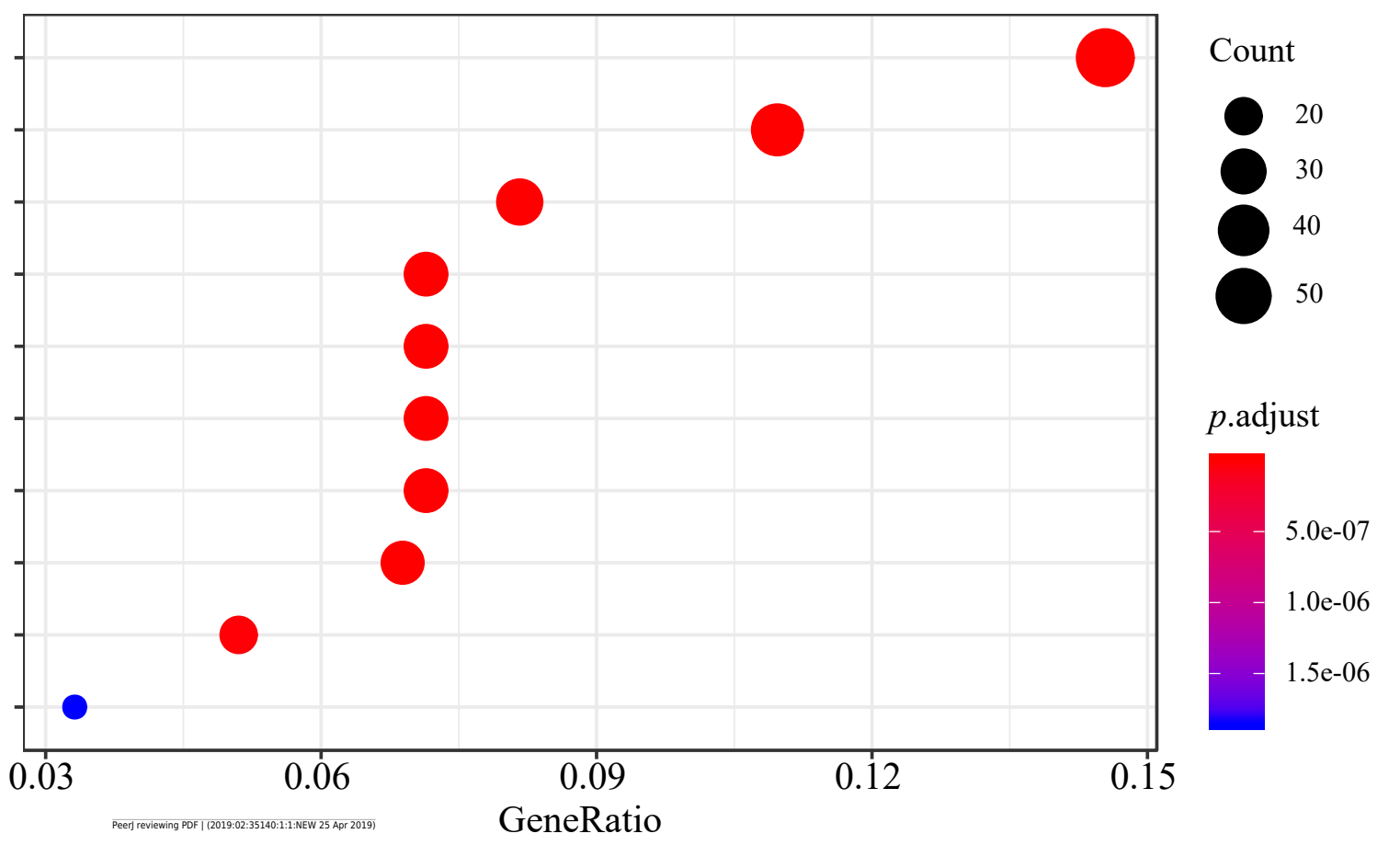


Figure $\mathbf{5}$ (on next page)

The most significant module in the PPI network of the DEGs.

The most significant module includes 528 edges and 33 nodes. The circles represent genes, and the lines represent interactions between the proteins encoded by the genes. 


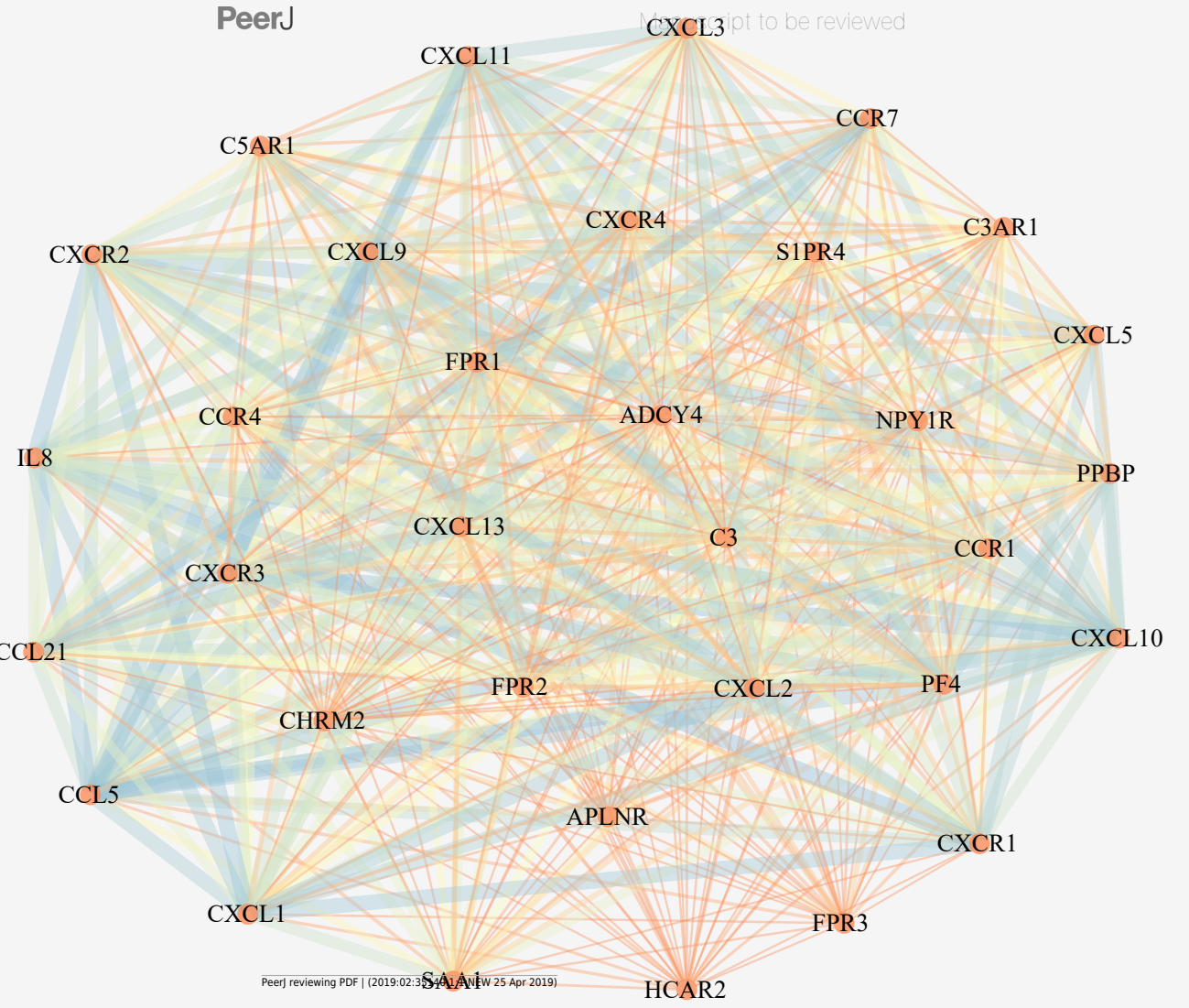




\section{Figure 6}

Construction of ceRNA network in pulpitis.

(A) Venn diagram showing the number of distinct and overlapping RNAs among the upregulated genes and the RNAs identified with miRDB, starBase, and TargetScan. The overlapping areas show the upregulated genes identified by three online tools. (B) Interaction of RNAs in the PVT1-associated ceRNA network. The triangle node and the diamond node represent the IncRNA and miRNA, respectively. The rectangle nodes represent miR-455-5ptargeted mRNAs. The round nodes are the top 10 hub DEmRNAs in the network. The up- and downregulated genes are colored in red and green, respectively.

A

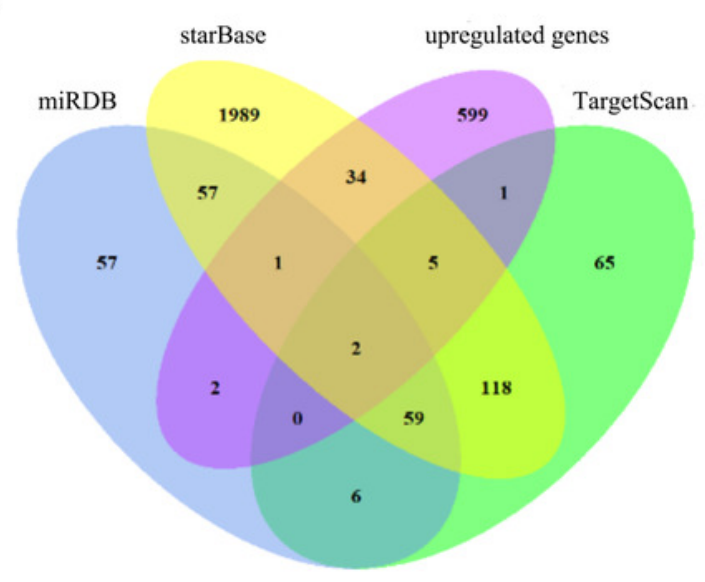

$\mathrm{B}$

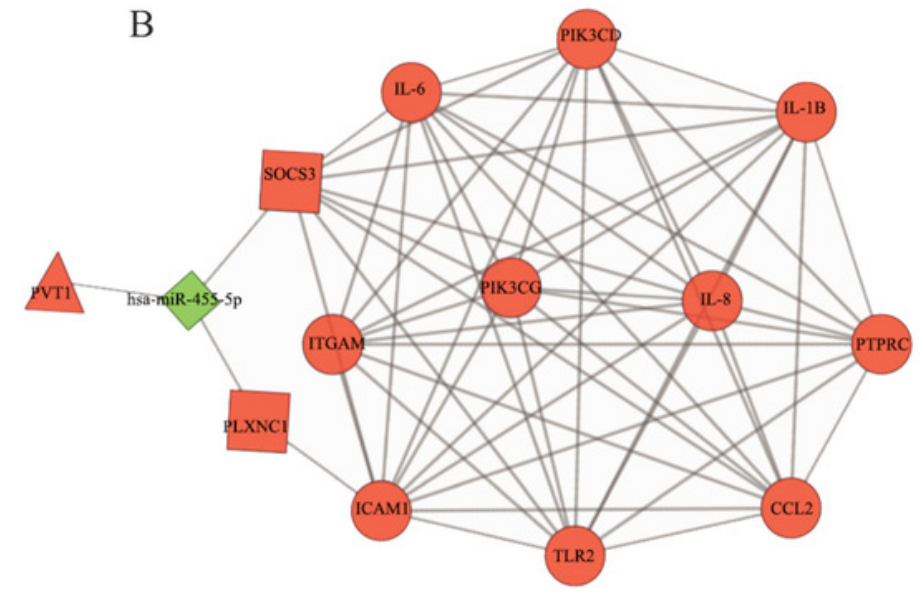


Figure 7 (on next page)

Relative expression levels of PVT1 in inflamed and normal pulp tissue.

The transcript levels of PVT1 were determined by qRT-PCR and normalized to those of the reference RNA GAPDH. $p$-value $=0.03$ 


\section{Manuscript to be reviewed
Wilcoxon, $\mathrm{p}$-value $=0.03$}

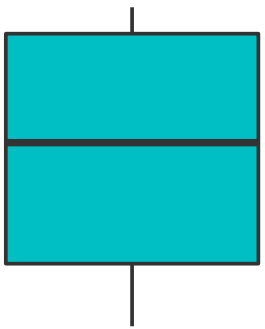


Table $\mathbf{1}$ (on next page)

Top 15 enriched GO terms of the DEGs. 
Table 1: Top 15 enriched GO terms of the DEGs.

2

\section{Terms}

GO.BP:0070098

GO.BP:0060326

GO.BP:0002690

GO.BP:0050921

GO.BP:0006954

GO.CC:0005615

GO.CC:0009897

GO.CC:0005886

GO.CC:0071944

GO.CC:0005887

GO.MF:0045236

GO.MF:0008009
Pathway description

chemokine-mediated signaling pathway

cell chemotaxis

positive regulation of leukocyte chemotaxis

positive regulation of chemotaxis

inflammatory response

extracellular space

external side of plasma membrane

plasma membrane

cell periphery

integral component of plasma membrane

CXCR chemokine receptor binding

chemokine activity
Count

17

21

17

18

23

15

6

19

19

10

11

13
$P$-Value

$4.46 \mathrm{E}-35$

$2.18 \mathrm{E}-33$

$2.84 \mathrm{E}-30$

$7.66 \mathrm{E}-30$

$5.00 \mathrm{E}-29$

1.79E-07

0.000948

0.00174

0.00174

0.0144

5.28E-25

2.39E-24 
GO.MF:0001664

GO.MF:0048248

GO.MF:0008528
G-protein coupled receptor binding

CXCR3 chemokine receptor binding

G-protein coupled peptide receptor activity
15

5

9
$8.21 \mathrm{E}-20$

4.67E-12

$2.18 \mathrm{E}-11$ 
Table 2 (on next page)

Top 5 enriched KEGG pathways of the DEGs. 
Table 2:Top 5 enriched KEGG pathways of the DEGs.

2

\begin{tabular}{llcc}
\hline Category & Pathway description & Count & P-Value \\
\hline KEGG:map04062 & chemokine signaling pathway & 21 & $1.49 \mathrm{E}-33$ \\
KEGG:map04060 & cytokine-cytokine receptor interaction & 20 & $4.10 \mathrm{E}-28$ \\
KEGG:map05150 & Staphylococcus aureus infection & 6 & $1.78 \mathrm{E}-08$ \\
KEGG:map04080 & neuroactive ligand-receptor interaction & 9 & $2.16 \mathrm{E}-08$ \\
KEGG:map04668 & TNF signaling pathway & 6 & $9.52 \mathrm{E}-07$ \\
\hline
\end{tabular}

